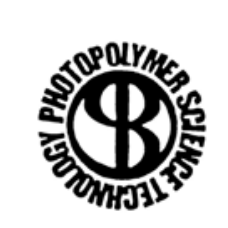

\title{
Electroless and Electrolytic Plating of Photopolymerized Resin for Use in the Micro-Molding of Three-Dimensional Nickel Structures
}

by

Kohki Mukai, Toshiya Yoshimura, Shinya Kitayama, and Shoji Maruo*

Department of Mechanical Engineering and Materials Science

Graduate School of Engineering, Yokohama National University,

79-5 Tokiwadai, Hodogaya, Yokohama, Kanagawa 240-0197, Japan

Reprinted from

Journal of Photopolymer Science and Technology

Vol. 20, No.2, 285-290 (2007) 


\title{
Electroless and Electrolytic Plating of Photopolymerized Resin for Use in the Micro-Molding of Three-Dimensional Nickel Structures
}

\author{
Kohki Mukai, Toshiya Yoshimura, Shinya Kitayama, and Shoji Maruo * \\ Department of Mechanical Engineering and Materials Science \\ Graduate School of Engineering, Yokohama National University, \\ 79-5 Tokiwadai, Hodogaya, Yokohama, Kanagawa 240-0197, Japan
}

\begin{abstract}
We investigated the electroless and electrolytic plating of photopolymerized resin for use in the molding of three-dimensional nickel micro-structures. The micro-molding process consists of four steps: fabrication of the resin mold, electroless plating of the mold, electrolytic grinding to open the interior structure, and extraction. An epoxy resin mold, which is suitable for two-photon micro-polymerization, was successfully plated with nickel through this electroless process. Electrolytic plating was found to be useful for applying a thick nickel film on non-conductive resin with low crystal lattice distortion and few impurities. Regarding the design of the mold opening, it was shown that electroless plating does not proceed inside non-through-holes on the sub-millimeter scale, but that it proceeds inside through-holes with a diameter of $550 \mu \mathrm{m}$ and down to the depth of $2 \mathrm{~mm}$.
\end{abstract}

Keyword: Keywords: electroless plating, electrolytic plating, microstructure, molding, photopolymerization, nickel

\section{Introduction}

Metal is not used much in the production of micro-machines due to its difficulty in micro-processing, in contrast to semiconductors, although its mechanical characteristics and special properties such as magnetism are attractive in a wide range of applications. Micro-fabrication with photopolymerized resin is regarded as one of the promising micro-part production technologies because of its suppleness, easiness and quickness [1-4]. However, due to the weakness of photopolymerized resin against environmental factors (e.g., high temperature, acidity and alkalinity), there are some limits to its practical use. To further develop the possibilities of the photo microfabrication technique, several studies have been carried out, and there have been reports on the combination of photopolymerization and electroless metal plating. For example, Formanek, et al. have fabricated a silvercoated micrometer-sized photopolymer structure aligned periodically on a glass surface [4]. But since these reported structures contain a polymer inside as the base, the weakness against environmental factors will still limit their use. In order to address this issue, three-dimensional metal microfabrication using photochemical eduction has been proposed [5]. However, the difficulty in controlling the reaction volume, which can only be controlled on the micron scale at best, limits the applicability of this

Received March 30, 2007

Accepted May 10, 2007 
technique as well.

We propose a novel micro-molding method for the fabrication of pure metal micro-structures [6,7]. Our micro-molding process consists of four major steps. First, raw resin is polymerized by scanning with a focused laser beam to form the three-dimensional negative shape of the microstructure, provided with a small opening. The resolution of the polymerization is as low as $100 \mathrm{~nm}$ when using the two-photon absorption method [2]. Next, the non-conductive polymer mold is coated with metal through electroless plating. In this step, the inner space of the negative shape is filled by the metal. Then, the obstructive metal is removed by electrolytic grinding to expose the opening of the mold. It should be noted that the electrolytic grinding stops by itself when the non-conductive resin is exposed, since the inner metal and outer coating are electrically isolated [8]. Thus, a precise cut is attained at the opening of the mold. Finally, the microstructure is extracted by removing the polymer. The removal is done by burning or melting the polymer through heating, or by dissolving the polymer through a chemical process. In our previous work, the electroless plating conditions for nickel, when used in conjunction with epoxy resin subjected to two-photon polymerization, were found, and the extraction of the nickel microstructure from the resinous mold was achieved.

In this paper, we investigated the electroless and electrolytic plating of photopolymerized resin for the molding of three-dimensional nickel micro-structures. The hybrid process of electrolytic and electroless plating was examined to determine the conditions under which the plated body becomes sufficiently thick, the process is sufficiently fast and the resin has good crystal properties. We also investigated the optimal diameter of the opening for electroless nickel plating, to aid in the design of the mold micro-structure.
2. Hybrid process of electroless and electrolytic plating

We examined the thickening and purity of plated nickel film created by means of a hybrid process of electroless and electrolytic plating. In order to fill the inner space of the mold, electrolytic plating is used once the electroless metal coating has been applied. This hybrid plating method will also aid in the fabrication multi-layered metal and metal alloy using thermal interdiffusion.

We used an epoxy resin suitable for two-photon polymerization. A cup made of silicone was first prepared, and the resin was polymerized in the cup using an ultraviolet (UV) lamp for the convenience of sample preparation. The sample was pellet-shaped and formed in the cup. The appearance of the sample before plating is shown in Fig. 1(a).

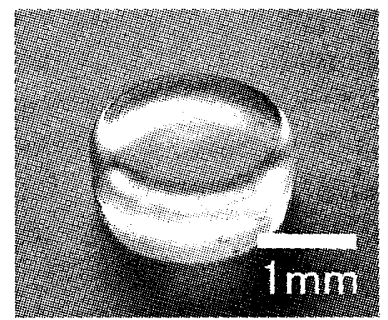

(a)

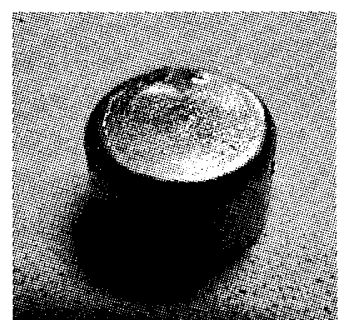

(b)
Figure 1 Pellet of resin (a) as polymerized, (b) and after nickel electroless plating.

Before the electroless plating, any contamination on the resin surface was rinsed out with pure water and acetone under ultrasonic waves. Then, the sample was immersed in the catalytic solution, composed of $\mathrm{PdCl}_{2}, \mathrm{SnCl}_{2}$ and $\mathrm{HCl}$ at $30^{\circ} \mathrm{C}$ for 5 minutes. Subsequently, the sample was immersed in the plating solution, composed of $\mathrm{NiSO}_{4}, \mathrm{C}_{2} \mathrm{H}_{5} \mathrm{O}_{2} \mathrm{~N}, \mathrm{PbO}$ and $\mathrm{NaH}_{2} \mathrm{PO}_{2}$. The plating solution was continuously stirred during plating. The temperature was $45^{\circ} \mathrm{C}$, and the $\mathrm{pH}$ was maintained at 7 using $\mathrm{HCl}$. The growth rate of plating film was about 2.5 
$\mu \mathrm{m} / \mathrm{h}$. The mold after the electroless plating process is shown in Fig. 1(b), where we can see that the mold has been completely plated by glossy nickel.

After the electroless nickel plating, we performed the electrolytic nickel plating in a Watt's bath solution $\left(\mathrm{NiSO}_{4} \quad 7 \mathrm{H}_{2} \mathrm{O}, \mathrm{NiCl}_{2}\right.$ $\left.6 \mathrm{H}_{2} \mathrm{O}, \mathrm{H}_{3} \mathrm{BO}_{3}\right)$. An electric current can be applied to the sample after having done the nickel plating once. The temperature of the Watt's bath was $45^{\circ} \mathrm{C}$ and the $\mathrm{pH}$ was maintained at 1.5 . The growth rate of the plated film was $60 \mu \mathrm{m} / \mathrm{h}$, which is equivalent to 24 times that in electroless plating.

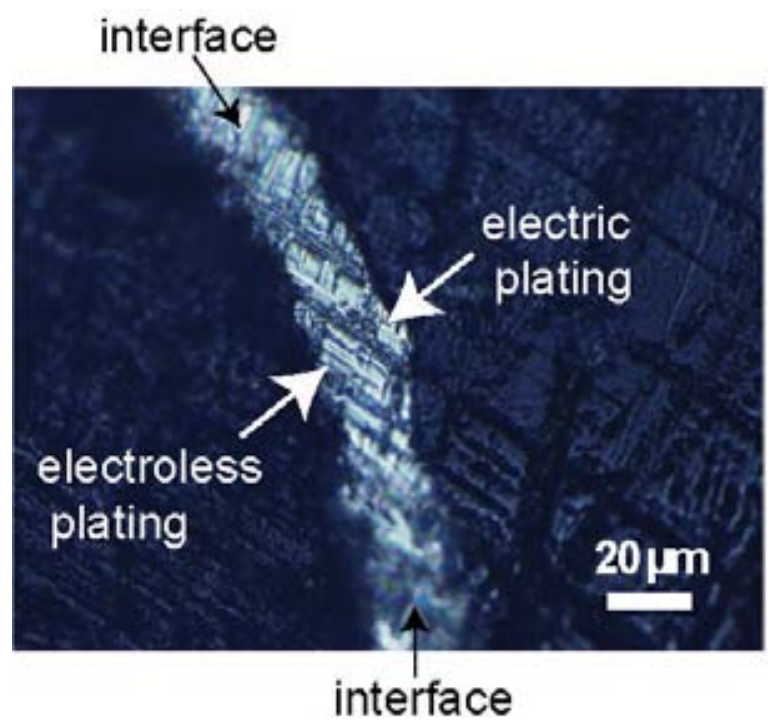

Figure 2 Cross-sectional optical microscope image of film plated through the hybrid process of electroless plating and electrolytic plating. For observation purposes, the film was supported by another plastic.

Fig. 2 shows a high-resolution optical microscope image of the cross-section of a plated film which was specifically applied to another plastic through a low-temperature process to enable microscope observation. In the figure, the electroless plating side is epoxy resin. The total film thickness is about $20 \mu \mathrm{m}$. The surface of the photopolymerized resin has many small pits $2-5 \mu \mathrm{m}$ in depth. This is because the surface of the resin replicated the surface of the silicone cup. Minute adherence of the plated metal to the polymer is required to fabricate micro-order structures. We see that the small pits are completely filled by the plated nickel, suggesting that the micrometer-size mold structure will be replicated by the nickel electroless plating. The border between the electrolytic plating and electroless plating is approximately in the middle of the film, but the border is not distinguishable by optical microscope observation. We can see that the coherency between the films is very good.

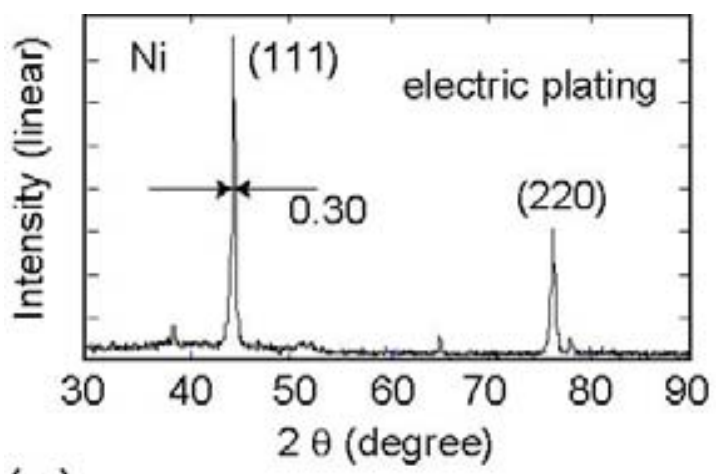

(a)

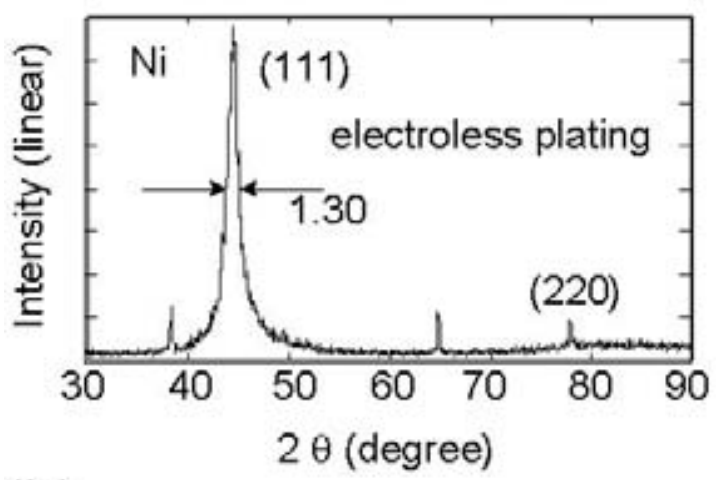

(b)

Figure 3 XRD curve of nickel formed by (a) electrolytic plating and (b) electroless plating. The FWHM of the $\mathrm{Ni}(111)$ peak was used to evaluate the crystal residual stress.

Fig. 3 shows the X-ray diffraction (XRD) spectra of the plated nickel film: 3(a) is for the electrolytic plating and $3(\mathrm{~b})$ is for the electroless plating. The positions of the diffraction peaks indicate the nickel metal. 
The broadening of the full width at half maximum (FWHM) of the diffraction peaks indicates the residual crystal stress. The FWHM of the strong Ni (111) peak of the electroplated film is $0.30^{\circ}$, less than $1 / 4$ that of the electroless-plated film $\left(1.30^{\circ}\right)$. This result suggests that the crystal of the electroplated film has fewer impurities and defects compared with that of the electroless-plated film. Note that impurities and dislocations generally accrue in the metal as the growth rate of plated film rises. Considering that the growth rate of electroplated film is higher by more than one order of magnitude than that of electroless-plated film, film formation by electrolytic plating is very effective.

Table I Components of the nickel film plated through the electroless and the electrolytic processes. "UDL" means "under the detection limit."

\begin{tabular}{ccc}
\hline (mass\%) & $\begin{array}{c}\text { electroless } \\
\text { plating }\end{array}$ & $\begin{array}{c}\text { electric } \\
\text { plating }\end{array}$ \\
\hline $\mathrm{Ni}$ & 92.8 & 95.87 \\
$\mathrm{Cl}$ & 0.29 & 1.681 \\
$\mathrm{P}$ & 4.3 & (UDL) \\
$\mathrm{Na}$ & 1.98 & (UDL) \\
$\mathrm{S}$ & (UDL) & 0.917 \\
\hline
\end{tabular}

We evaluated the component elements of the plated nickel film by X-ray fluorescence measurement. The results are listed in Table $I$. Here, we compared the components of the films formed by the electroless plating and electrolytic plating. Impurities were attributed to the catalytic solution and the plating solution. The purity of the electroless-plating nickel film was more than $90 \%$, but that of the electroplating nickel film was even higher. The result agrees well with the FWHM presented in Fig. 3.

The results presented in this section clearly suggest that the hybrid process of electroless and electrolytic plating is very useful for obtaining high-quality metal micro-structures in a short processing time.

\section{Electroless plating through a small opening \\ We examined the possibility of} electroless plating inside minute structures. The continuous and uniform supply of metal ions to the surface is necessary to prevent voids in plating. This will determine the design of the mold.

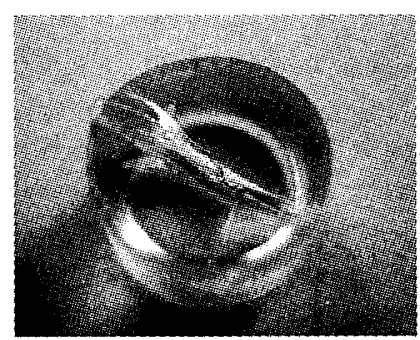

(a)

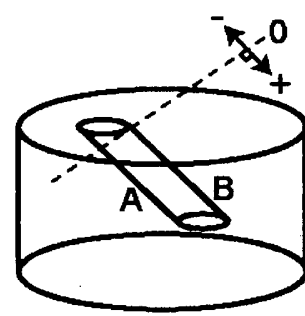

(b)
Figure 4 (a) Photograph of a resin sample with a non-through-hole. (b) A schematic view of a sample with a non-through-hole and the definition of the position inside the hole.

We prepared a resin piece with a non-through-hole $1 \mathrm{~mm}$ in diameter and $3 \mathrm{~mm}$ deep, and performed electroless plating. The hole was formed by dipping a metal stick into the raw resin during photopolymerization. Vacuuming was performed during soaking the sample into the plate solution so that plate solution entered the hole thoroughly, with no bubbles remaining in the aperture. The plating solution was continuously stirred during plating. Fig. 4(a) shows a photograph of a resin pellet with a hole before plating. Fig. 4(b) schematically defines sides A and B and the distance from the opening.

Fig. 5 shows the relationship between the distance from opening and the plate film thickness. It can be seen that thickness of the 
plated film decreases with the depth of the aperture. The rate of decline on side $A$ is $-8.9 \% / \mathrm{mm}$ and that on side $B$ is $-10.9 \% / \mathrm{mm}$. These values are not very different, and the decline lines have a similar tendency. A film nearly $30 \mu \mathrm{m}$ in thickness was formed on one part at a depth of $2.5 \mathrm{~mm}$. In addition, we found the formation of a thick plating film at the bottom of the hole.

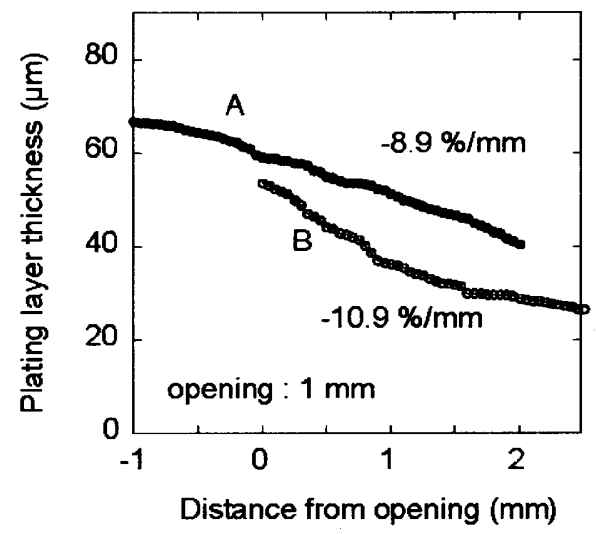

Figure 5 Plating layer thickness in a non-through-hole as a function of the distance from the 1-mm opening.

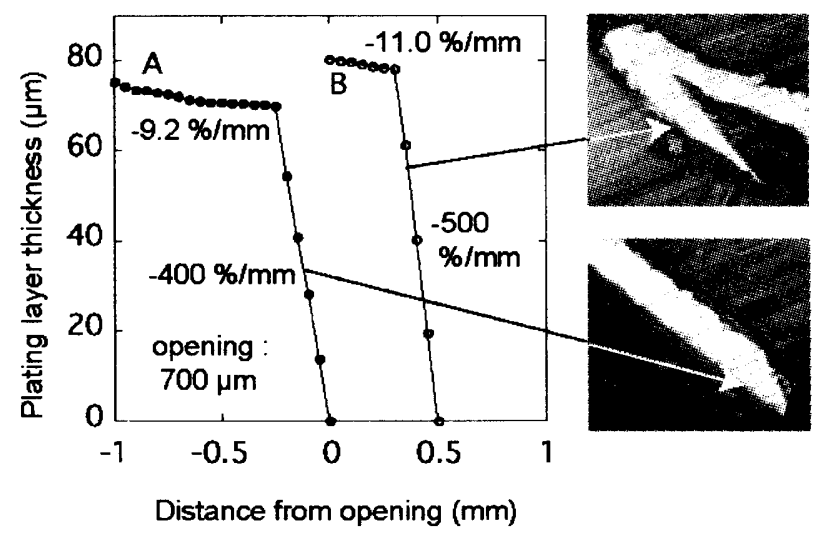

Figure 6 Plating layer thickness in a non-through-hole as a function of the distance from the $700-\mu \mathrm{m}$ opening. The sample cross-section was observed under an optical microscope.

Next, we prepared a resin piece with a non-through-hole $700 \mu \mathrm{m}$ in diameter and 3 $\mathrm{mm}$ in depth, and performed electroless plating. Fig. 6 shows the relationship between the distance from the opening and the plating film thickness. Two cross-section optical microscope photographs of the film are shown. A sudden decrease of the film thickness was observed at about $0.3 \mathrm{~mm}$ from the opening. The decline rate of the film thickness before the sudden decrease was almost the same as those shown in Fig. 5. Then, the decline rate increased to $-400 \sim-500 \% / \mathrm{mm}$. The reason for this sudden decrease is thought to be that the plating solution did not circulate through the inside of the aperture and the ion supply dried up in that region.

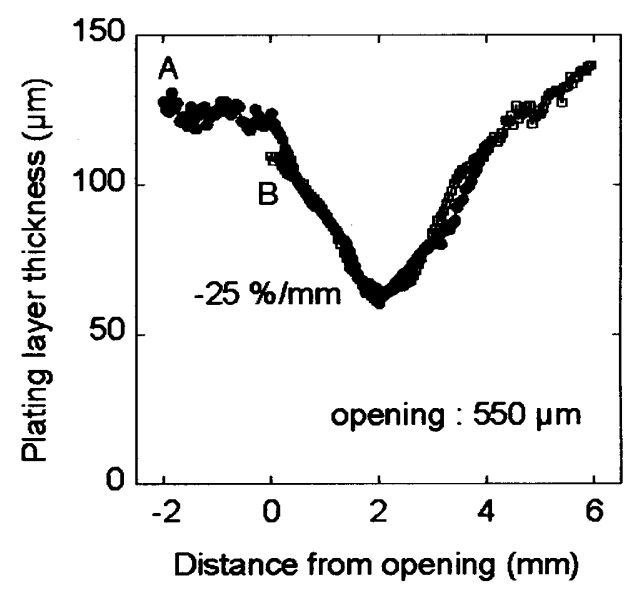

Figure 7 Plating layer thickness in a through-hole as a function of the distance from the $550-\mu \mathrm{m}$ opening.

We performed a similar experiment with a through-hole $550 \mu \mathrm{m}$ in diameter. Fig. 7 shows the relationship between the distance from the opening and the plating film thickness. It can be seen that the electroless plating proceeded from both ends of the aperture to the depth of $2 \mathrm{~mm}$, even though the aperture diameter was smaller than the $700 \mu \mathrm{m}$ in Fig. 6. The film thickness was as great as $50 \mu \mathrm{m}$ in the thinnest place. This result suggests that electroless plating can be used for producing small three-dimensional structures if a penetration-type mold structure is created to allow the plating solution to circulate easily. To ensure the proper 
circulation of the plating solution, the position and number of openings will be an important design parameter in addition to the size of the openings.

The results presented in this section distinctly indicate that the size of the mold opening on the sub-millimeter scale is crucial for the circulation of the plate solution inside the closed space of the mold.

\section{Conclusion}

We investigated the electroless and electrolytic plating of photopolymerized resin for the molding of three-dimensional nickel micro-structures. The electrolytic plating process was successfully hybridized with the electroless plating process. This hybrid method was found to be effective in speeding up the plating, reducing the crystal residual stress and improving the purity of the nickel metal films. We also determined the size of the opening that is required in the design of molds for electroless plating. It was shown that electroless plating does not proceed inside a non-through-hole on the sub-millimeter scale, but that it proceeds inside a through-hole with a diameter of 550 $\mu \mathrm{m}$ down to the depth of $2 \mathrm{~mm}$.

\section{Acknowledgement}

This work was supported by Electro Mechanic Technology Advancing Foundation.

\section{References}

[1] S. Maruo and S. Kawata, J. Microelectromech. Syst. 7 (1998) 411.

[2] S. Kawata, H-B. Sun, T. Tanaka and K. Takada, Nature 412 (2001) 697.

[3] K. Nishimura, H. Uchida, M. Inoue, M. Sendoh, K. Ishiyama and K. I. Arai, J. Appl. Phys., 93 (2003) 6712.

[4] F. Formanek, N. Takeyasu, T. Tanaka, K. Chiyoda, A. Ishikawa and S. Kawata, Appl. Phys. Lett., 88 (2006) 083110.

[5] T. Tanaka, A. Ishikawa and S. Kawata, Appl. Phys. Lett. 88 (2006) 081107.

[6] T. Yoshimura, S. Maruo, and K. Mukai, Ext. Abs. Int. Conf. Solid State Devices and Materials 2006 (Yokohama, Japan).

[7] K. Mukai, T. Yoshimura, and S. Maruo, Jpn. J. Appl. Phys. in press (2007).

[8] Toshikazu Sato, "Denkaikakou to Kagakukakou (Electrolytic Processing and Chemical Processing)" (Asakura Shoten, Tokyo, 1970) (in Japanese). 